BMJ Open

Diabetes

Research

\& Care

\section{Factors influencing the effect of mindfulness-based interventions on diabetes distress: a meta-analysis}

To cite: Guo J, Wang H, Luo J, et al. Factors influencing the effect of mindfulness-based interventions on diabetes distress: a meta-analysis. BMJ Open Diab Res Care 2019;7:e000757. doi:10.1136/ bmjdrc-2019-000757

- Additional material is published online only. To view please visit the journal online (http://dx.doi.org/10.1136/ bmjdrc-2019-000757).

Received 17 July 2019 Revised 18 September 2019 Accepted 9 November 2019

Check for updates

(C) Author(s) (or their employer(s)) 2019. Re-use permitted under CC BY-NC. No commercial re-use. See rights and permissions. Published by BMJ.

${ }^{1}$ Xiangya School of Nursing, Central South University, Changsha, Hunan, China ${ }^{2}$ School of Life Science, Central South University, Changsha, Hunan, China

${ }^{3}$ School of Foreign Languages, Central South University, Changsha, Hunan, China ${ }^{4}$ Department of Family and Community Medicine and Institute for Health Policy Research, University of California, San Francisco, California, USA

${ }^{5}$ School of Nursing, Yale University, New Haven, Connecticut, USA

Correspondence to Dr Jia Guo;

guojia621@163.com

\section{ABSTRACT}

To review the evidence and determine the factors influencing the effect of mindfulness-based interventions (MBI) on diabetes distress. A systematic search of nine databases (PubMed, Cochrane Library, Web of Science, PsycINF0, Embase, China Knowledge Resource Integrated, VIP Data, SinoMed Data, and Wan Fang Data) was conducted. Randomized controlled trials of MBls for adults with diabetes that evaluated the effect of the interventions on diabetes distress were retrieved. Meta-analysis was conducted by using Review Manager V.5.3, a Cochrane Collaboration tool. Subgroup analyses were conducted for exploring factors influencing the effect of MBIs on diabetes distress. A total of 10 articles, consisting of eight studies with 649 participants, were included. The results from subgroup analyses on the studies revealed five factors that influenced the effect of MBls on diabetes distress compared with control group. Participants with elevated baseline diabetes distress showed a moderate effect size of 0.48 of decreasing diabetes distress when receiving MBIs $(p=0.005)$; the MBls based on mindfulness-based stress reduction therapy alleviated diabetes distress of the participants with a large effect size of $0.58(p<0.0001)$; the MBls delivered in group format decreased the diabetes distress with a moderate effect size of $0.36(p=0.03)$; the MBIs with home practice assignment alleviated the diabetes distress with a moderate effect size of 0.42 $(p=0.05)$. The long-term rather than short-term effect of MBIs on diabetes distress reduction has been identified with large effect size of $0.56(p=0.04)$. MBls improve outcomes in adults with diabetes who have elevated diabetes distress at baseline, using mindfulness-based stress reduction therapy, using a group format to deliver the intervention, and assigning home practice. MBls improve diabetes distress significantly more at long-term follow-up compared with short-term follow-up. MBls could be considered as an adjunct treatment in adults with diabetes to reduce diabetes distress.

\section{INTRODUCTION}

Diabetes is a major health problem worldwide due to its rapidly growing prevalence and high disease burden. It is a major cause of blindness, renal failure, cardiovascular disease and lower limb amputation. ${ }^{1}$ The prevalence of diabetes is predicted to grow to 642 million by 2040 , and it is anticipated to be the seventh leading cause of death by $2030 .^{2}$ Diabetes costs exceeded US $\$ 727$ billion in 2017 , and contributes to approximately $12 \%$ of the total medical expenses for adults worldwide. ${ }^{3}$ Research has shown that living with diabetes is challenging. In the face of the complex and demanding daily self-management, adults with diabetes may become frustrated, angry, overwhelmed, and/or discouraged.$^{45}$ Psychological comorbidity is high in people with diabetes, with extensive research demonstrating that approximately $30 \%$ of adults experience depressive symptoms. ${ }^{6}$ Diabetes distress is another psychological disorder among adults with diabetes, with a slightly higher prevalence compared with depressive symptoms in one study ( $36 \%$ vs $30 \%){ }^{7}$

Diabetes distress refers to negative emotions in response to living with diabetes (eg, feeling frustrated, hopeless, angry, guilty, fearful), which has been reported to occur in $18 \%-45 \%$ of adults with diabetes. ${ }^{8-10}$ Diabetes distress is exacerbated by lack of understanding of diabetes self-management, unhelpful interactions with family, friends and health professionals, and feeling overwhelmed by the demands of managing the condition 511 Diabetes distress has been associated with less self-management, poor glycemic control ${ }^{12}$ and low health-related quality of life ${ }^{13-15}$ Diabetes distress is not associated with clinical depression or anxiety, ${ }^{16} 17$ and is less recognized and treated in clinical care compared with anxiety and depression. ${ }^{18}$

There are several interventions aimed to reduce psychological comorbidity, such as diabetes distress in adults with diabetes, including cognitive-behavioral therapy, problem-solving therapy, network-based cognitive-behavioral therapy, and mindfulness therapy. ${ }^{19}$ Overall, compared with conventional diabetes education, these interventions can effectively relieve diabetes distress and show moderate beneficial effects 
on depression, anxiety, and general psychological distress. ${ }^{20}$ Mindfulness-based interventions (MBI) have been increasingly used to alleviate negative emotions such as stress, anxiety, depression, and diabetes distress among adults with diabetes. ${ }^{21} 22$ MBIs can not only help adults with diabetes learn to cope with distress without escaping the stressful emotion, thus preventing or delaying physiological complications. ${ }^{23-25}$ MBIs can also contribute to better self-care and self-management behaviors. ${ }^{21}$

MBIs are derived and adapted from Buddhist practices to help individuals relax their minds and achieve a state of calmness, peace, and happiness. Breathing techniques and meditation exercises are used, aiming to channel non-judgmental attention into the present moment. ${ }^{26}$ Research on the effect of MBIs on health has exponentially increased in the past decade. ${ }^{27}$ There are several different principles of mindfulness therapies, which include mindfulness-based stress reduction (MBSR), mindfulness-based cognitive therapy (MBCT), acceptance and commitment therapy, dialectical behavior therapy (DBT), and mindfulness-based self-compassion. The different approaches of these mindfulness therapies are displayed in online supplementary Appendix 1. MBIs that have been evaluated in adults with diabetes have focused on MBSR and MBCT.

Recently, a systematic review and meta-analysis was conducted on the effect of MBIs on quality of life, diabetes distress, and glycemic control in adults with diabetes. ${ }^{21}$ MBIs demonstrated a small-to-moderate effect size for pretreatment to post-treatment changes in diabetes distress and metabolic control among treatment group participants. However, in the eight studies included in this systematic review, there were clinical and methodological heterogeneity in baseline diabetes distress levels of adults, the principles of MBIs, the intervention delivery (group vs individual), the use of home practice, and length of follow-up. The purpose of this systematic review was to explore the influence of these factors on the effect of MBIs on diabetes distress using subgroup analysis. The evidence synthesized can then be used to help guide future research and clinical practice in the use of MBIs for adults with diabetes.

\section{METHODOLOGY}

This study was conducted according to Preferred Reporting Items for Systematic Reviews and Meta-Analyses guidelines for systematic reviews and meta-analyses, ${ }^{28}$ including a systematic search, inclusion/exclusion criteria specification, evaluation of study quality, data extraction, and data analysis. A systematic review protocol was developed in July 2018 which included information on the background of the population and intervention of interest, proposed search strategies, eligibility criteria, selection process, data management and extraction, quality assessment, and data analysis. Throughout the review, the protocol was used as a guide in searching and managing eligible studies.

\section{Search strategies}

A systematic search strategy was developed in consultation with a medical librarian. Nine databases were searched: PubMed, Cochrane Library, Web of Science, PsycINFO, Embase, China Knowledge Resource Integrated, VIP Data, SinoMed Data, and Wan Fang Data. Keywords and Medical Subject Headings (MeSH) were used as part of the search strategy with the MeSH heading 'Diabetes' expanded for associated subheadings. For Chinese electronic databases, the search terms included 'Mindfulness' and 'Diabetes'. For English electronic databases, search terms included ('diabetes mellitus') OR 'diabetes' AND 'mellitus' OR 'diabetes mellitus' OR 'diabetes' OR ('diabetes') AND distress AND ('mindfulness') .

The reference lists of retrieved articles were also hand searched to locate any additional studies not included in the database search results. We did not restrict by year of publication. A preliminary search was performed on 22 August 2018 and the final search was performed on 24 January 2019. The complete search strategy is provided in online supplementary appendix 2.

\section{Inclusion and exclusion criteria}

We included studies that met the following inclusion criteria: (A) adults with type 1 or type 2 diabetes; (B) evaluation of MBIs; (C) reported outcome of diabetes distress; (D) randomized controlled trials (RCT); and (E) reported findings in English or Chinese. We excluded studies that (A) did not include measurement of diabetes distress; or (B) had missing, incomplete or unclear data that were required for the meta-analysis. ${ }^{29}$

\section{Data selection}

Two authors (JG, HW) independently assessed abstracts and titles for eligibility and excluded articles that did not meet the inclusion criteria. If it was unclear whether an article met the inclusion criteria, a full-text review was completed. Consensus was achieved with all included studies.

Data extracted from studies included: (A) study characteristics (authors, country, year of publication, sample size, and mean age, gender, race/ethnicity of participants); (B) intervention strategy (principles of MBIs, MBI delivery format, length, dosage, interventionist, and setting); (C) diabetes distress measurement (diabetes distress tool and time points of measurement); and (D) efficacy evaluation (mean and SD of diabetes distress evaluated at all time points in both groups of each study to generate the effect size). If there were two control groups in one study, for example, walking for one control group and diabetes education for the other control group ${ }^{30}$ the data from the diabetes education group were extracted as the control group to conduct the analysis, because the majority of control group interventions were diabetes education, thus avoiding heterogeneity. 


\section{Risk of bias assessment}

Risk of bias for each study was assessed independently by two researchers using the risk of bias tool outlined in the Cochrane Handbook for Systematic Reviews of Interventions ${ }^{31}$ The tool includes six key criteria for potential risk of bias: adequacy of allocation sequence generation; adequacy of allocation concealment; blinding of adults, personnel or outcome assessors; completeness of outcome data; selectivity of outcome reporting, and other biases. The two reviewers settled any disparities by consulting the third independent reviewer and any consensus were documented.

\section{Data synthesis}

Data were analyzed using the Review Manager software (RevMan V.5.3, Cochrane Collaboration, Oxford, UK). All data were double entered into the database to minimize error. In a preliminary analysis, descriptive statistics of individual variables and characteristics of included studies were examined. Second, effect sizes representing the standardized mean difference between MBIs and control groups or between before and right after intervention of all the RCTs were estimated. When the subgroup analysis was conducted to compare the diabetes distress results of different evaluation time points with the control group, all available data were extracted. Effect sizes of less than 0.2 can be interpreted as small, those in the range of $0.2-0.5$ are moderate, and effect sizes of greater than 0.5 are considered large. ${ }^{32}$

Heterogeneity was estimated using Cochran's Q test and $\mathrm{I}^{2}$ statistics. The statistical significance of heterogeneity was $\mathrm{p}<0.10$, and the degree of variability was estimated through $\mathrm{I}^{2}$ values, with $75 \%, 50 \%, 25 \%$, or $0 \%$ indicating high, moderate, low, or no heterogeneity, respectively. ${ }^{33}$
The fixed effects model was used in the absence of any significant heterogeneity ( $p$ value of $Q$-test $>0.10$ and $\mathrm{I}^{2}$ value $<50 \%$ ), while the random effects model was used if heterogeneity was significant ( $p$ value of $Q$-test $<0.10$ and $\mathrm{I}^{2}$ value above $50 \%$ value but below $\left.75 \%\right) .{ }^{34}$ In this review, subgroups were based on the following intervention characteristics: baseline diabetes distress levels of participants, the principles of the MBIs, delivery format (group vs individual), and the assignment of home practice. The effect of the intervention at short-term and long-term follow-ups (3 and 6 months) on diabetes distress reduction was also compared between groups.

\section{RESULTS}

\section{Study selection}

The search yielded 270 articles (figure 1). After removing 109 duplicates, 161 articles remained. The titles and abstracts of these articles were screened for inclusion/ exclusion, resulting in 34 potential articles of interest. After reading the full texts of these articles, 22 were excluded because diabetes distress was not measured and two articles were removed because the required outcome data (diabetes distress score) were unavailable. A total of eight studies (from 10 articles) met the inclusion criteria.

\section{Risk of bias}

The risk of bias summary is presented in figure 2. Eight studies were rated as low risk of selection bias because they all reported random sequence generation process. ${ }^{30} 35-41$ Four of the eight studies $(50 \%)$ were rated as low risk of allocation concealment, reporting allocation conducted by an independent statistician, a trained researcher, research nurse, or a file with password protection. ${ }^{30} 353640$

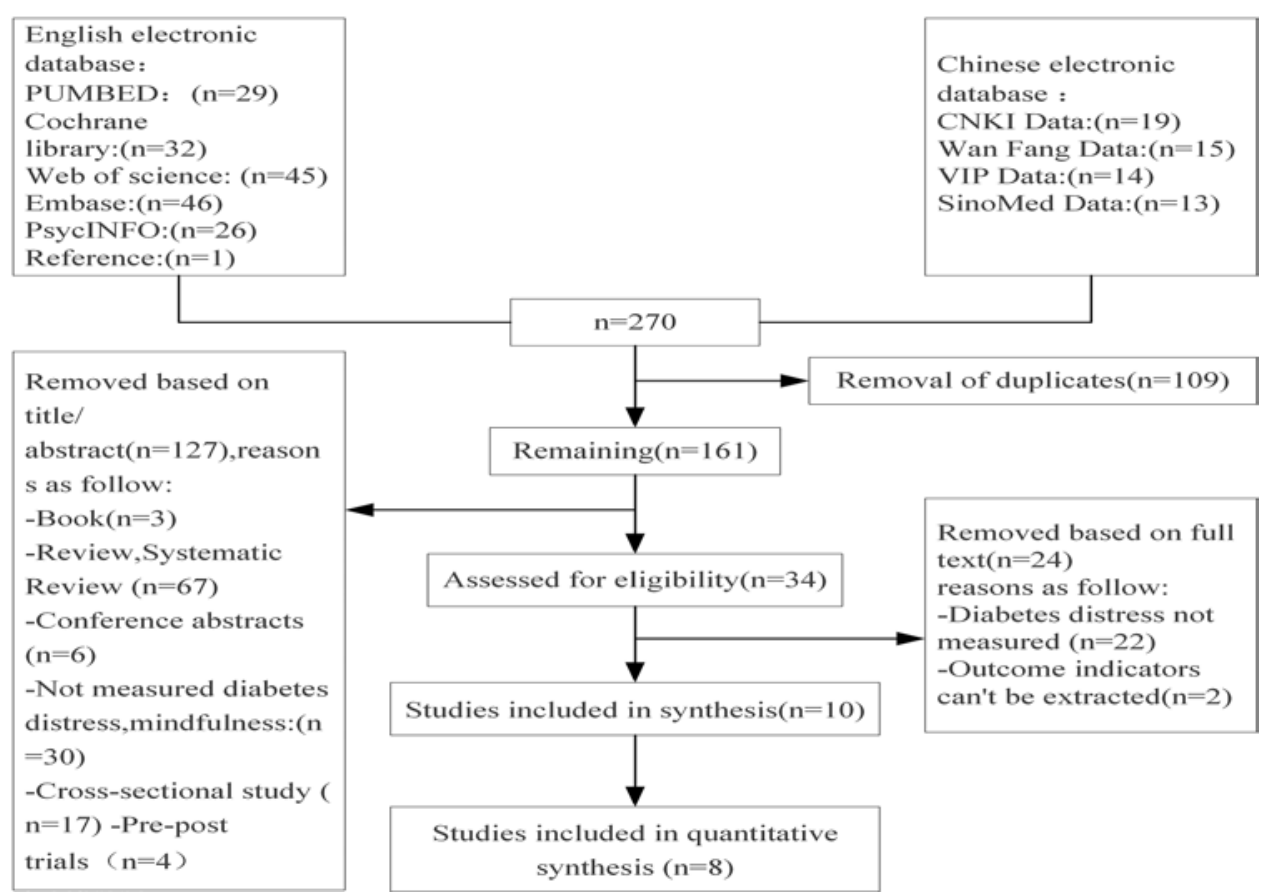

Figure 1 Summary of literature search. 


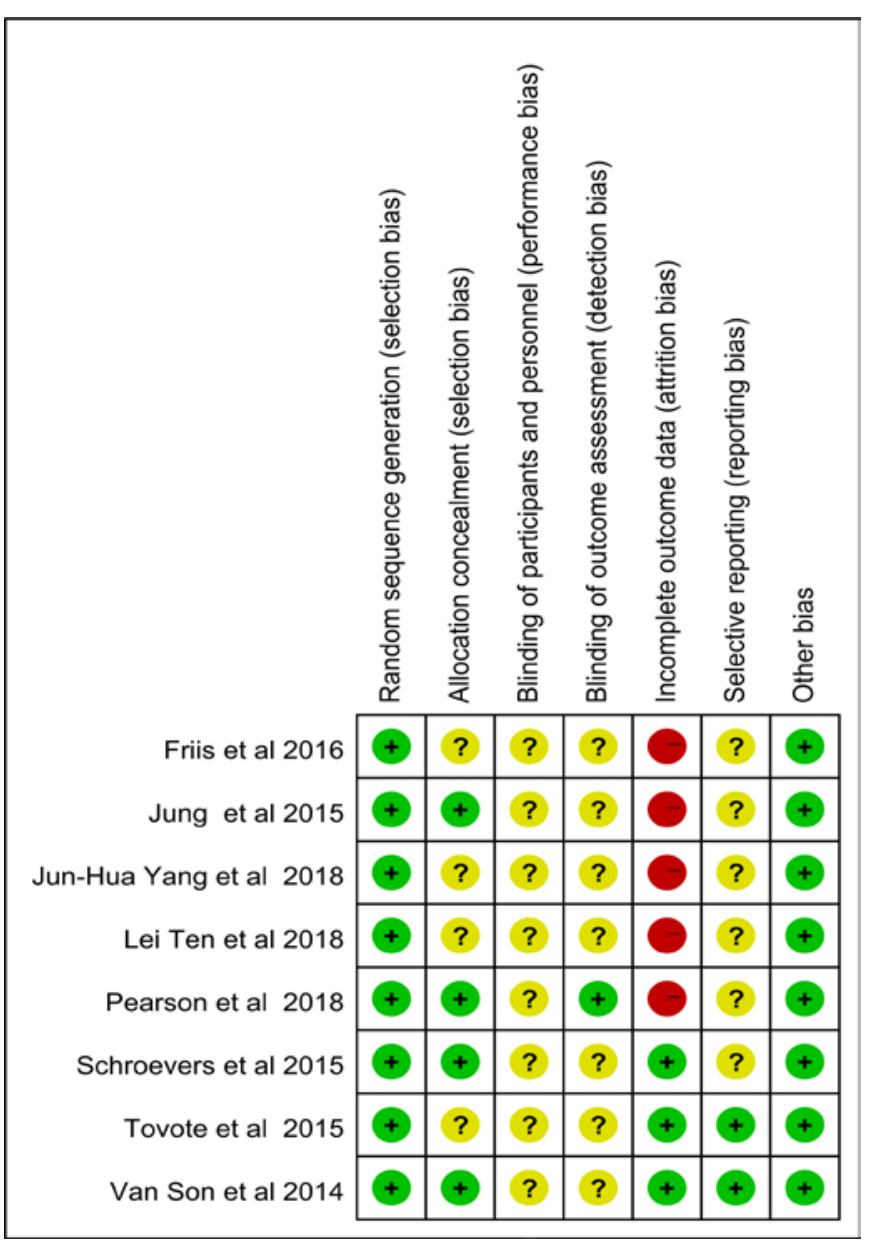

Figure 2 Risk of bias according to the Cochrane risk of bias tool.

All eight studies were rated as unclear risk of blinding of adults and personnel bias, because the process was not applicable for MBIs. ${ }^{30}{ }^{35-41}$ Only one study (12.5\%) was rated as low risk of blinding of outcome assessment bias, ${ }^{40}$ because the data were collected by a research nurse who did not know the details of group allocation. Intentionto-treat analysis was reported in three studies, ${ }^{35-37}$ indicating low risk of incomplete outcome data bias. Two of the studies $(25 \%)$ had published a protocol and reported all prespecified outcomes, thus were rated as low risk of reporting bias. ${ }^{35} 37$

\section{Study characteristics}

Eight studies were included in this meta-analysis, with a total of 649 adults from six countries including Australia $(n=1)$, China $(n=2)$, Netherlands $(n=3)$, New Zealand $(n=1)$, and South Korea $(n=1)$. The total sample size of the MBI groups was 293, ranging from 12 to 70 per group, while the total sample size of control groups was 312, ranging from 12 to 69 per group. All studies were published in journal articles from 2014 to 2018, with $75 \%$ of studies $(\mathrm{n}=6)$ reported in the years 2015 and 2018, respectively ${ }^{30}{ }^{36} 37{ }^{39-41}$ Six studies were published in English journals ${ }^{30} 35-3840$ and two studies were published in Chinese journals. ${ }^{39} 41$
There was a wide age range of adults, from 18 to 70 years old. The mean age of participants in the MBI group across all studies ranged from 42 to 67 years old, and the mean age ranged from 46 to 68 years old in the control group across all studies. Four studies consisted of a mixed type 1 /type 2 diabetes populations with type 1 diabetes accounting $30 \%-73 \%$ of the sample ${ }^{35-38}$ and four studies included only adults with type 2 diabetes. ${ }^{30}{ }^{39-41}$

Two validated measures of diabetes distress were used in studies evaluating the impact of MBIs. Four used the Problem Areas in Diabetes Scale (PAID) ${ }^{35-37} 40$ and four used the Diabetes Distress Scale (DDS).$^{30-41}$ Although the DDS has a stronger focus on motivational and behavioral problems associated with diabetes self-management and the PAID covers a greater variety of emotional concerns (including diabetes-related emotional burnout and diabetes non-acceptance), the two scales have overlapping content, have similar psychometric properties and are similarly correlated with a variety of criterion measurements. ${ }^{42}$

At baseline, there were five studies reporting an elevated diabetes distress of participants (above criterion scores with DDS-17 and PAID-20, respectively) in both intervention and control groups. ${ }^{30} 36383941$ There were three studies reporting mean diabetes distress of participants at baseline below the criterion score for increased diabetes distress. ${ }^{35} 3740$ The characteristics of studies included in this review are provided in table 1 .

\section{The interventions}

\section{The MBIs}

There were various principles of MBIs among the eight studies, including MBCT $(\mathrm{n}=3),{ }^{35-37}$ MBSR $(\mathrm{n}=3),{ }^{30}{ }^{41}$ DBT $(\mathrm{n}=1),{ }^{40}$ and mindfulness-based selfcompassion $(\mathrm{n}=1) .{ }^{38}$

The majority of the MBIs included six to eight sessions for 8 weeks $(n=7) .^{30}{ }^{35-40}$ A 2-hour booster session was added 3 months after the end of the intervention as a means to boost MBIs ${ }^{35}$ There is one MBI that included a 2-week program, with three sessions per week, six sessions in total. ${ }^{41}$ Across all interventions, each session lasted 20-180 min with the majority of sessions lasting 30-60 min. MBIs were delivered one-on-one $(n=4)$ or in a group setting $(n=4)$.

There were four MBIs that included a home practice assignment, ${ }^{35-3741}$ while others did not assign any home practice $(n=4) .{ }^{30}{ }^{38-40}$ The dosage of the home practice assignment was about $30 \mathrm{~min} /$ day $(\mathrm{n}=2)$, with a length of 7-8 weeks. ${ }^{35-37}$ The content of the home practice assignments included performing a body scan, mindful eating exercises, routine activity with awareness, or a short sitting meditation on breath. ${ }^{35-3741}$

The interventionists providing the MBIs included psychologists $(n=4)^{35-38}$ and a multidisciplinary team of healthcare providers and psychologists $(n=1) .{ }^{41}$ In two studies, the interventionist of the MBIs was not reported. ${ }^{30} 39$ The majority of studies were conducted in hospital clinics $(n=5), 36-3941$ with one MBI conducted in a community 


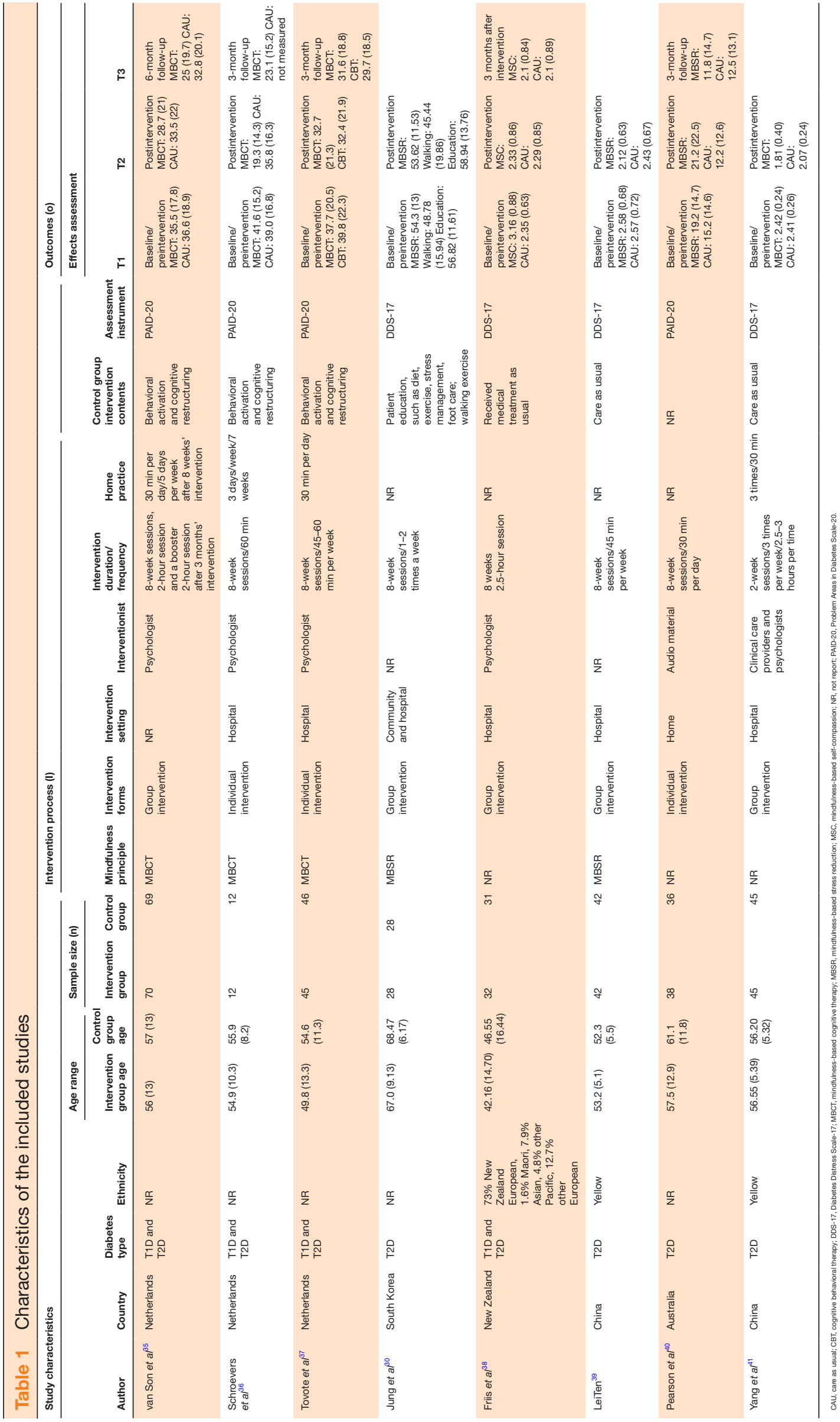


healthcare center and hospital ${ }^{30}$ and the other MBIs conducted at home whereby the participants were asked to follow all the MBI sessions by audio materials at home, without any in-person interventions. ${ }^{40}$ There was one study which did not report intervention setting. ${ }^{35}$

The attrition of the MBIs that used an MBSR approach intervention arm ranged from $7 \%$ to $25 \%$, and control arms were $7 \%-39 \%$. The attrition of MBIs that used an MBCT approach intervention arm ranged from $17 \%$ to $71 \%$, and control arms were $0 \%-22 \%$. In addition, the attrition of the MBIs that used a mindful self-compassion approach intervention arm was $14 \%$, and control arm was $19 \%$. Reasons for attrition included schedule conflicts or not interested in participating. ${ }^{30} 3538$

\section{The control group}

In four studies, the control group received diabetes education (eg, the definition of diabetes, a description of symptoms, self-management strategies, and medication)..$^{30}$ 38-41 In three studies, the control group received psychological counseling, such as behavioral activation and cognitive restructuring. ${ }^{35-37}$ In one study, the details of the control group were not reported. ${ }^{40}$

\section{The measurement of diabetes distress and the evaluation time points}

In the majority of studies, data collection was completed on completion of the intervention $(n=7) \cdot{ }^{3035-40}$ There were three studies that reported 3-month follow-up effect. ${ }^{36} 3740$ There were two studies reporting data collection at 6 months after baseline. ${ }^{3541}$
The influencing factors of the effect of the MBIs on diabetes distress

Subgroup analyses were conducted to examine the influencing factors on the efficacy of the MBIs, including baseline diabetes distress level (above criterion scores vs below criterion scores), the principles of MBIs (MBSR vs MBCT), MBI delivery (group vs individual), the use of home practice, and efficacy evaluation time points (right after MBIs, after 3 months, and after 6 months). The results of betweengroup comparisons of factors influencing the effect of MBIs on diabetes distress are provided in online supplementary appendix 3 .

\section{Comparison of MBI efficacy on baseline diabetes distress level among adults}

There was a statistically significant decrease of diabetes distress with a moderate effect size in the MBI group compared with the control group when studies reported an increased diabetes distress at baseline (above criterion scores) $(\mathrm{n}=5) \quad($ effect size $=-0.48$, $95 \%$ CI -0.81 to $-0.15, \mathrm{Z}=2.82, \mathrm{p}=0.005)$. There was no statistical significance on diabetes distress between the intervention and control groups when studies reported a normal average diabetes distress at baseline $(n=3) \quad$ (effect size $=0.05,95 \%$ CI -0.34 to 0.44 , $\mathrm{Z}=0.24, \mathrm{p}=0.81$ ) (see figure 3 ).

\section{Comparison on the efficacy of MBIs with different principles} on diabetes distress

There was a statistically significant decrease of diabetes distress in the MBI group with a moderate effect size compared with the control group when

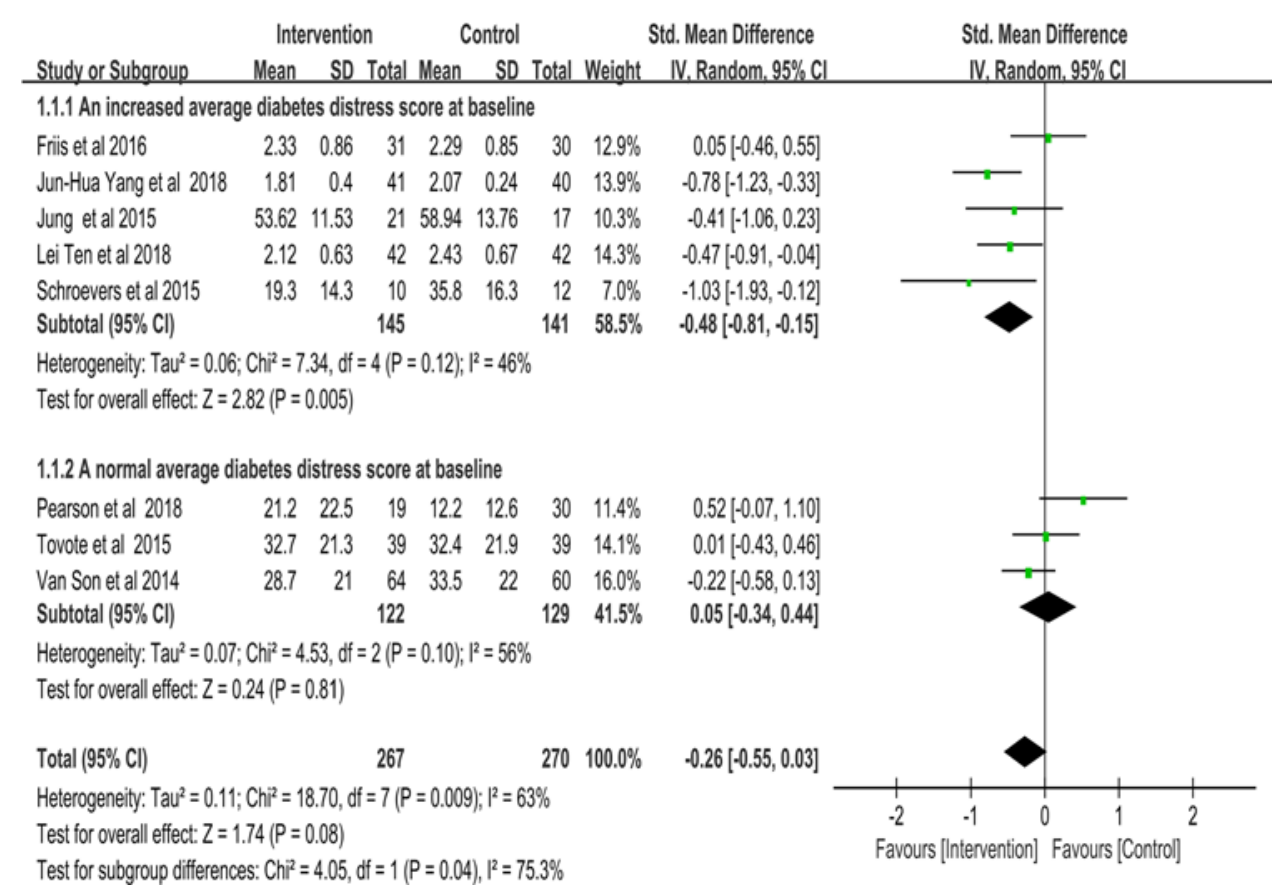

Figure 3 Forest plot: effectiveness of mindfulness-based interventions (MBI) on diabetes distress among adults with an above cut-off diabetes distress versus below cut-off diabetes distress at baseline. 


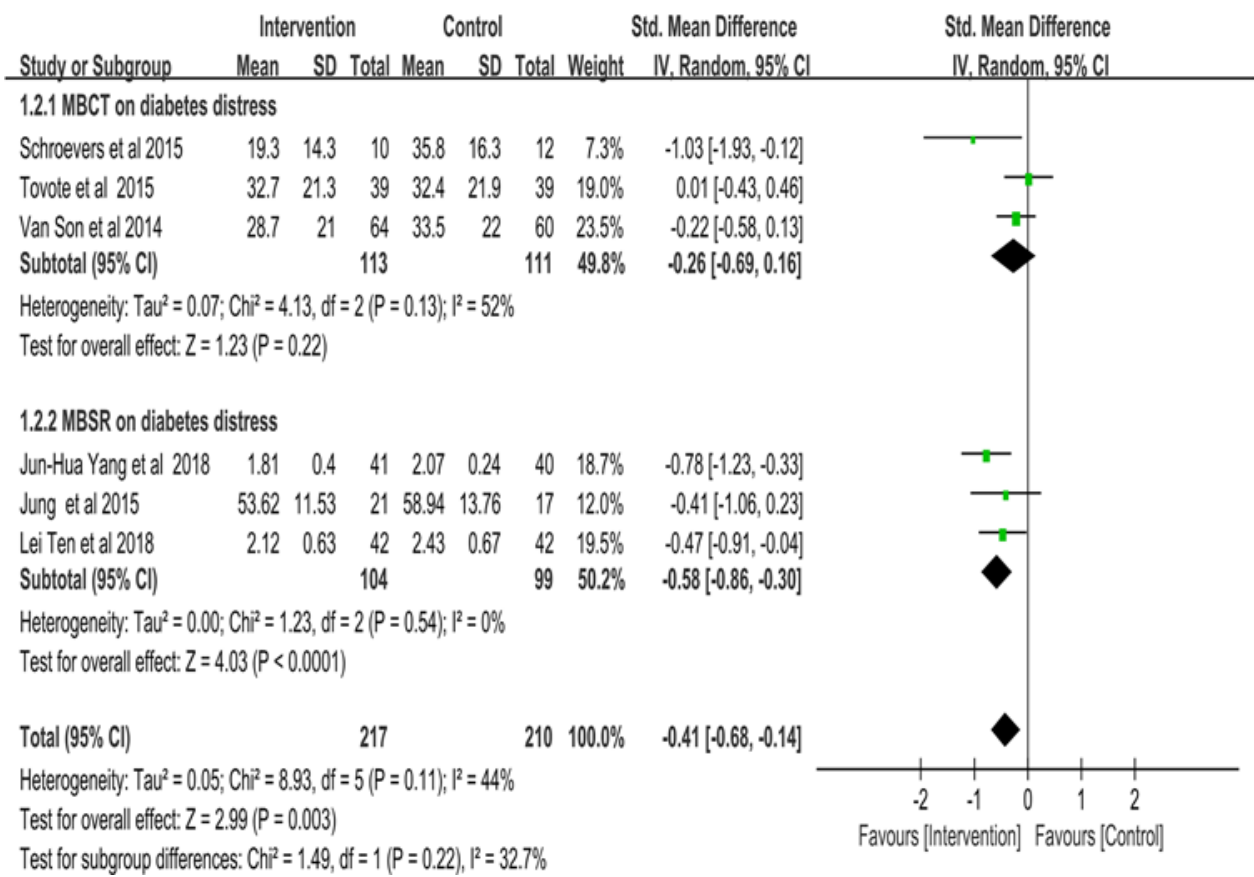

Figure 4 Forest plot: effectiveness of mindfulness-based stress reduction/mindfulness-based cognitive therapy (MBSR/ $\mathrm{MBCT}$ ) on diabetes distress.

MBSR was used as the principle of the MBIs $(n=3)$ (effect size $=-0.58,95 \%$ CI -0.86 to $-0.30, \mathrm{Z}=4.03$, $\mathrm{p}<0.0001)$. There was no statistically significant difference on diabetes distress between the MBI group and the control group when MBCT was used as the principle of the MBIs $(n=3)$ (effect size $=-0.26,95 \%$ CI -0.69 to $0.16, \mathrm{Z}=1.23, \mathrm{p}=0.22$ ) (see figure 4 ).

\section{Comparison on the efficacy of MBIs with different delivery} formats on diabetes distress among adults with diabetes

There was a statistically significant decrease of diabetes distress in the MBI group with a moderate effect size compared with the control group when the MBIs were delivered in a group format $(n=4)$ (effect size $=-0.36$, $95 \%$ CI -0.68 to $-0.04, \mathrm{Z}=2.20, \mathrm{p}=0.03$ ). There was no statistically significant difference between the MBIs and control group when the MBI was delivered in one-to-one format $(n=4) \quad($ effect size $=-0.15,95 \%$ CI -0.70 to 0.40 , $\mathrm{Z}=0.55, \mathrm{p}=0.58$ ) (see figure 5 ).

\section{Comparisons on the efficacy of MBIs with or without home practice assignment on diabetes distress among adults with diabetes}

There was a statistically significant decrease of diabetes distress in the MBI group with a moderate effect size compared with the control group when MBIs included a home practice assignment $(\mathrm{n}=4)$ (effect size $=-0.42,95 \%$ CI -0.84 to $-0.00, \mathrm{Z}=1.98, \mathrm{p}=0.05)$. When a home practice assignment was not included in the MBIs, there was no statistically significant difference on diabetes distress between the two groups $(\mathrm{n}=4)$ (effect size $=-0.09,95 \%$ CI -0.54 to $0.35, \mathrm{Z}=0.40, \mathrm{p}=0.69$ ) (see figure 6 ).

\section{Comparison on the effects of MBls when diabetes distress} was measured at different time points

There was no statistically significant difference of diabetes distress in the MBI group compared with the control group when the immediate effect of the intervention was measured $(\mathrm{n}=7)$ (effect size $=-0.17,95 \%$ CI -0.45 to $0.11, \mathrm{Z}=1.18, \mathrm{p}=0.24$ ). There was no statistically significant difference of diabetes distress in the MBI group compared with the control group when the effect was evaluated at 3-month follow-up $(\mathrm{n}=3) \quad$ (effect size $=0.02$, $95 \%$ CI -0.27 to $0.31, \mathrm{Z}=0.15, \mathrm{p}=0.88$ ). However, there was a statistically significant decrease of diabetes distress in the MBI groups with a large effect size compared with the control group when the effect at 6-month follow-up was evaluated $(\mathrm{n}=2) \quad($ effect size $=-0.56,95 \%$ CI -0.94 to $-0.18, \mathrm{Z}=2.91, \mathrm{p}=0.004$ ) (see figure 7).

\section{DISCUSSION}

In this meta-analysis, available evidence on factors affecting the effectiveness of MBIs in alleviating diabetes distress was evaluated, including baseline diabetes distress level, delivery format, type of MBIs, and length of follow-up. Eight studies involving MBIs were included. The age distribution was primarily middle-aged and elderly participants, with a much smaller number of young people with diabetes. In $50 \%$ of the studies, the MBIs were delivered by psychologists.

In the subgroup analyses, we found that adults with diabetes distress above the criterion score for elevated diabetes distress at baseline had significantly reduced diabetes distress after receiving the MBIs compared with the control group. Thus, MBIs are most effective in 


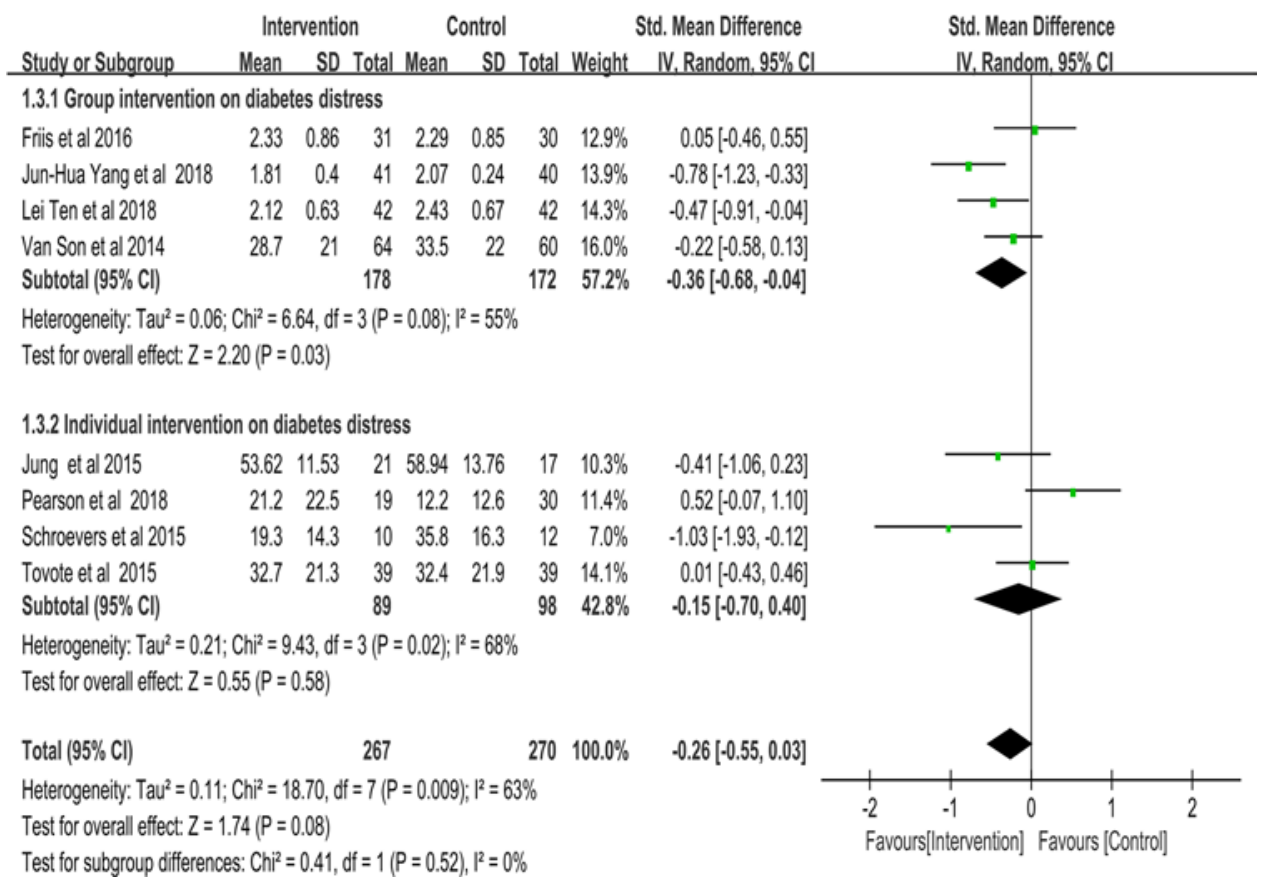

Figure 5 Forest plot: effectiveness of mindfulness-based interventions (MBI) with different delivery formats on diabetes distress.

reducing diabetes distress in those with elevated distress at baseline which is consistent with the results of a metaanalysis on the effect of the MBIs among adults with chronic illness on generalized anxiety disorder, depression, and other psychiatric or medical conditions. ${ }^{43}$ MBIs may also be able to help prevent an increase in diabetes distress in those with levels below criterion score; thus preventing more severe distress which influences selfmanagement, glycemic control, and quality of life.
The type of MBIs also appears to have an effect on reducing diabetes distress in adults with diabetes. MBSR therapy demonstrated a large effect on reducing diabetes distress, which is consistent with the results of a metaanalysis on the effects of MBSR on depression, anxiety, and psychological distress demonstrating a small effect size among adults with chronic illness.${ }^{44}$ However, MBCT did not improve diabetes distress outcomes compared with the control group in this analysis, possibly because

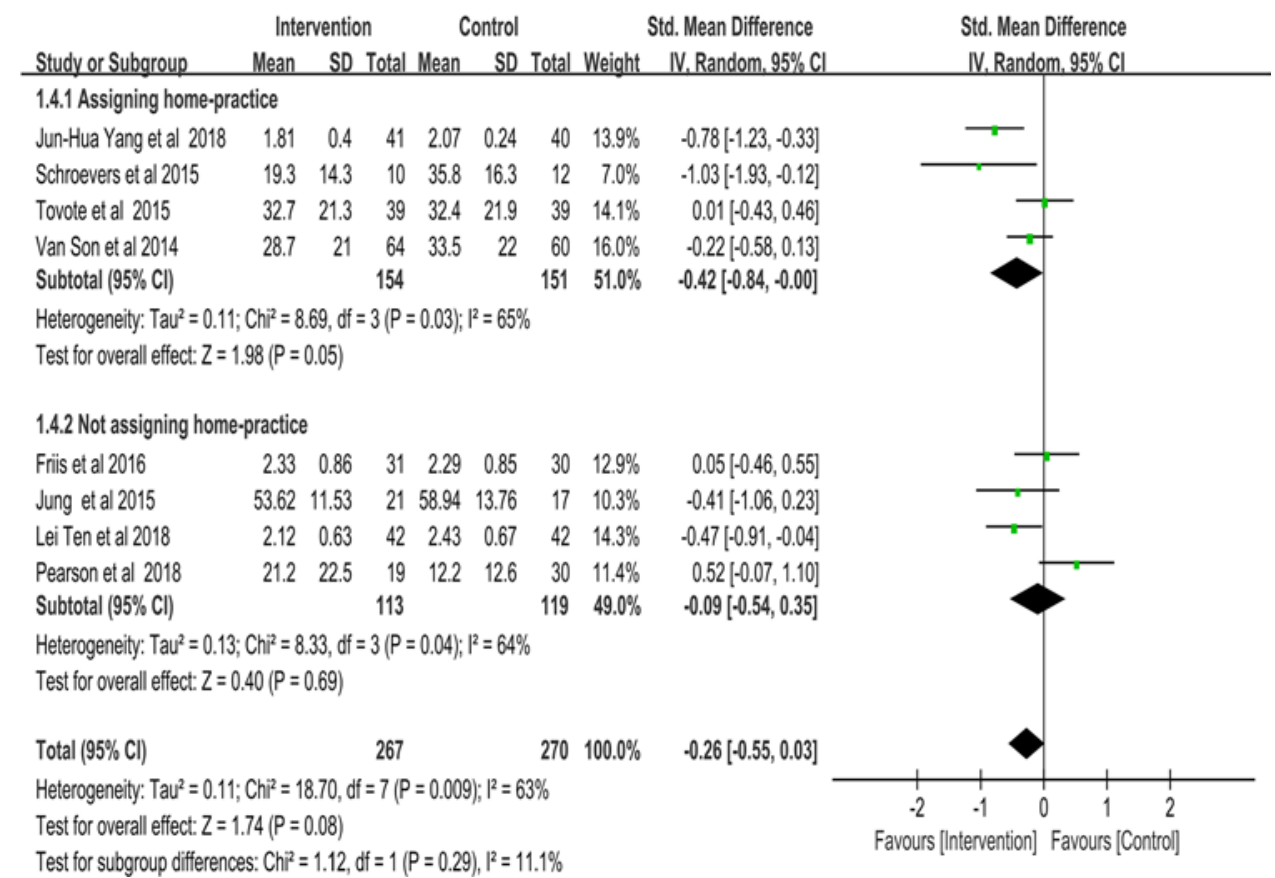

Figure 6 Forest plot: effectiveness of mindfulness-based interventions (MBI) with or without home practice assignment on diabetes distress. 


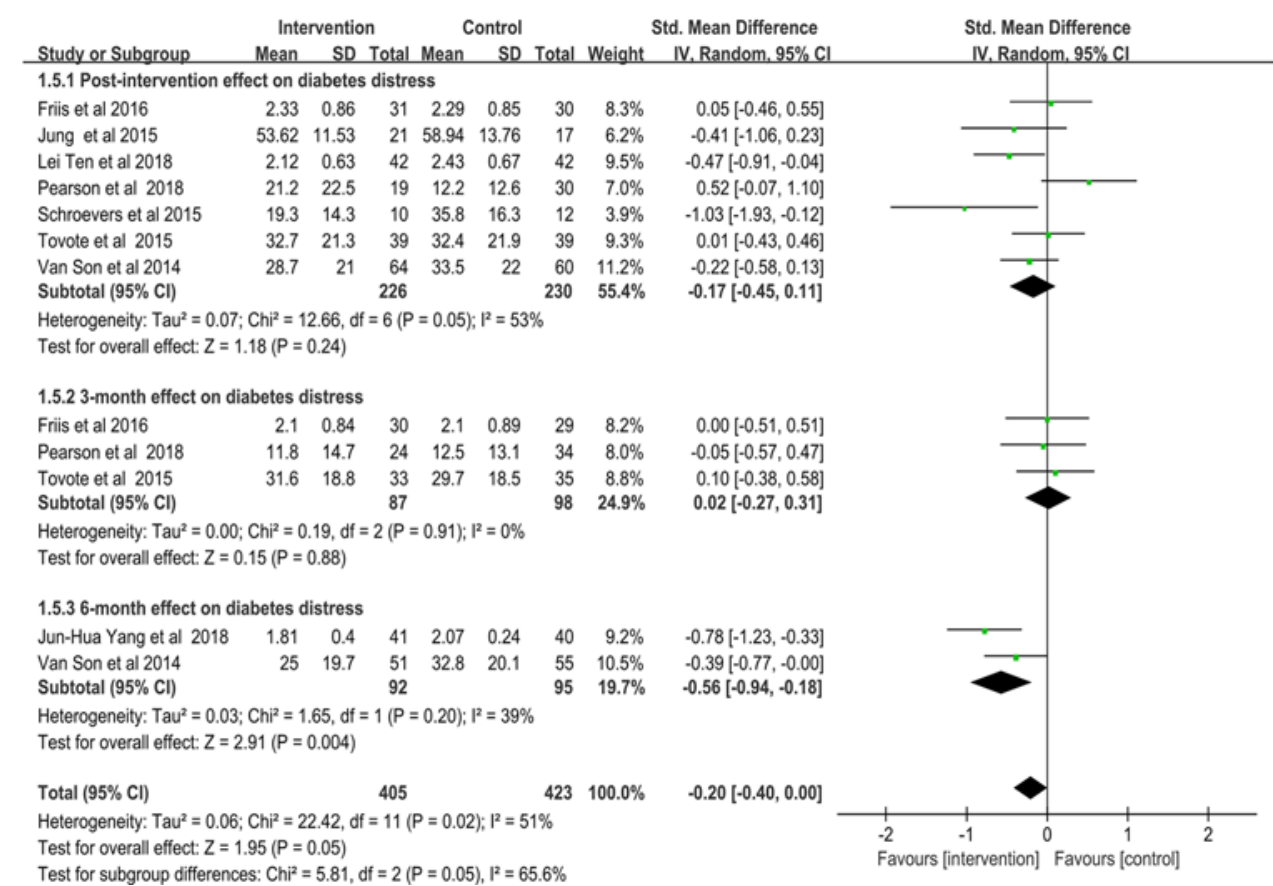

Figure 7 Forest plot: effectiveness of postintervention/3-month/6-month effect of mindfulness-based intervention on diabetes distress.

MBCT was designed for people with a history of recurrent depression to help learn how to disengage from depression-related and ruminative thoughts in order to prevent future episodes of depression. ${ }^{45}$ Thus, MBSR may be more effective in alleviating diabetes distress than MBCT according to the different mechanism of the treatment.

Group-based MBIs were more effective in reducing diabetes compared with other delivery formats. Structured group-based MBIs that included various modalities such as MBSR and MBCT were also found beneficial across a range of psychological and psychosocial issues encountered by individuals with vascular disease. ${ }^{46}$ Group psychotherapy is a well-established strategy for the treatment of depression, bipolar disorder, and anxiety disorder. ${ }^{47}$ In group psychotherapy, participants can learn vicariously through other group members, gain insight into themselves from multiple perspectives, obtain support, and find solutions through supportive group discussion.$^{48}$

Assigning formal home practice was also more effective in alleviating diabetes distress compared with programs that did not encourage home practice. These results are consistent with a systematic review of 43 MBI studies, whereby home practice besides MBI sessions demonstrated a small positive effect on reducing depression and anxiety in adults with cancer and insomnia.$^{49}$ The aim of home practice assignments is to help participants sustain regular mindfulness practices and integrate the practice into their daily schedules, providing more opportunity to achieve a state of calmness, peace, and happiness. Participants may also need time and practice to develop the MBI skills learnt during the intervention. This is also aligned with our finding that MBIs demonstrated stronger effects at longer follow-up compared with immediately after the intervention and at 3 months. Therefore, MBIs may take some time to integrate into daily life in order to alleviate diabetes distress.

\section{Strengths and limitations}

A strength of this meta-analysis was that only RCTs were included in this review, which are the gold standard to evaluate the effectiveness of the intervention study and provide the high-quality evidence. ${ }^{50}$ Overall, studies overall were of moderately good quality. Although the methodology for this systematic review was rigorous, some published or unpublished studies may not have been identified with our search strategies. Only English and Chinese publications were included, and research published in other languages on the effect of MBIs on diabetes distress may have been conducted. In some of our subgroup analyses, there were small sample subgroups (eg, 6-month follow-up). Thus, our subgroup comparisons are suggestive of factors that determine the efficacy of MBIs, but they do not provide definitive estimates in a multivariate context.

\section{Implication for practice and research}

There are several implications for clinical practice. First, screening for diabetes distress, in addition to depression and anxiety, may be important to consider in clinical practice. Subsequently, adults with increased diabetes distress may benefit from MBIs. Screening participants for elevated diabetes distress (above criterion score) using MBSR with group delivery format and home practice assignment are recommended. There are small 
available numbers of RCTs $(n=8)$, thus more RCTs in the field of MBIs and diabetes distress are indicated to contribute to the meta-analysis work. More research is needed on providing interventions in evaluating implementation of MBIs in the clinical setting using different health providers (eg, nurses), and investigating the longterm effects of the MBIs to alleviate diabetes distress.

\section{CONCLUSIONS}

This meta-analysis provides further evidence to support the use of MBIs in reducing diabetes distress in adults with diabetes. Factors influencing the effect of MBIs on diabetes distress have been identified, including diabetes distress status at baseline, group-based interventions, and using an MBSR therapy. Incorporating home practice and long-term follow-up also appears warranted.

Acknowledgements The authors express sincere gratitude to Xin Dong (Master, RN, Xiangya School of Nursing, Central South University) for his work on the bias evaluation of the included studies on this manuscript.

Contributors JG designed the study, obtained the grant and drafted the manuscript. HW assisted in data selection, data synthesis and drafting the manuscript. YG assisted in searching studies. JL and BL assisted in risk of bias assessment. YX and JW helped with the English language editing. RW conceptualized the study and critically reviewed the manuscript. All authors approved the final manuscript as submitted. JG is the guarantor of this work and has full access to all the data in the study and takes responsibility for the integrity of the data and the accuracy of the data analysis.

Funding This research was supported by the Philosophy and Social Science Foundation of Hunan Province (grant number: 17XBQ111) and Innovation-Driven Project of Central South University (grant number: 2018CX037).

Competing interests None declared.

Patient consent for publication Not required.

Provenance and peer review Not commissioned; externally peer reviewed.

Data availability statement All data relevant to the study are included in the article or uploaded as supplementary information.

Open access This is an open access article distributed in accordance with the Creative Commons Attribution Non Commercial (CC BY-NC 4.0) license, which permits others to distribute, remix, adapt, build upon this work non-commercially, and license their derivative works on different terms, provided the original work is properly cited, appropriate credit is given, any changes made indicated, and the use is non-commercial. See: http://creativecommons.org/licenses/by-nc/4.0/.

ORCID iD

Jia Guo http://orcid.org/0000-0002-8304-9947

\section{REFERENCES}

1 World Health Organization. Diabetes, 2017. Available: http://www. who.int/news-room/fact-sheets/detail/diabetes [Accessed 30 May 2019].

2 Mathers CD, Loncar D. Projections of global mortality and burden of disease from 2002 to 2030. PLoS Med 2006;3:e442.

3 International Diabetes Federation Annual Report. IDF diabetes atlas eighth edition, 2017. Available: https://www.idf.org/aboutdiabetes/ what-is-diabetes/facts-figures.html

4 Perrin NE, Davies MJ, Robertson N, et al. The prevalence of diabetes-specific emotional distress in people with type 2 diabetes: a systematic review and meta-analysis. Diabetic Medicine 2017;34:1508-20.

5 Polonsky WH, Fisher L, Earles J, et al. Assessing psychosocial distress in diabetes: development of the diabetes distress scale. Diabetes Care 2005;28:626-31.

6 Egede LE, Ellis C. Diabetes and depression: global perspectives. Diabetes Res Clin Pract 2010;87:302-12
7 Perrin N, Bodicoat DH, Davies MJ, et al. Effectiveness of psychoeducational interventions for the treatment of diabetesspecific emotional distress and glycaemic control in people with type 2 diabetes: a systematic review and meta-analysis. Prim Care Diabetes 2019.

8 American Diabetes Association. Standards of Medical Care in Diabetes--2014. Diabetes Care 2014;37:S14-80.

9 Fisher L, Polonsky WH, Hessler DM, et al. Understanding the sources of diabetes distress in adults with type 1 diabetes. $J$ Diabetes Complications 2015;29:572-7.

10 Pouwer F, Wijnhoven HAH, Ujcic-Voortman JK, et al. Ethnic aspects of emotional distress in patients with diabetes - the Amsterdam Health Monitor Study. Diabetic Med 2013;30:e25-31.

11 Pallayova M, Taheri S. Targeting diabetes distress: the missing piece of the successful type 1 diabetes management puzzle. Diabetes Spectrum 2014;27:143-9.

12 Adriaanse MC, Pouwer F, Dekker JM, et al. Diabetes-Related symptom distress in association with glucose metabolism and comorbidity: the Hoorn study. Diabetes Care 2008;31:2268-70.

13 Aikens JE. Prospective associations between emotional distress and poor outcomes in type 2 diabetes. Diabetes Care 2012;35:2472-8.

14 Fisher L, Hessler D, Glasgow RE, et al. REDEEM: a pragmatic trial to reduce diabetes distress. Diabetes Care 2013;36:2551-8.

15 Graue M, Haugstvedt A, Wentzel-Larsen T, et al. DiabetesRelated emotional distress in adults: reliability and validity of the Norwegian versions of the problem areas in diabetes scale (paid) and the diabetes distress scale (DDS). Int J Nurs Stud 2012;49:174-82.

16 Fisher L, Glasgow RE, Mullan JT, et al. Development of a brief diabetes distress screening instrument. Ann Fam Med 2008;6:246-52.

17 Fisher L, Mullan JT, Arean P, et al. Diabetes distress but not clinical depression or depressive symptoms is associated with glycemic control in both cross-sectional and longitudinal analyses. Diabetes Care 2010;33:23-8.

18 Pouwer F, Beekman ATF, Lubach C, et al. Nurses' recognition and registration of depression, anxiety and diabetes-specific emotional problems in outpatients with diabetes mellitus. Patient Educ Couns 2006;60:235-40.

19 Schmidt CB, van Loon BJP, Vergouwen ACM, et al. Systematic review and meta-analysis of psychological interventions in people with diabetes and elevated diabetes-distress. Diabet Med 2018;35:1157-72.

20 Rosenzweig S, Reibel DK, Greeson JM, et al. Mindfulness-based stress reduction is associated with improved glycemic control in type 2 diabetes mellitus: a pilot study. Altern Ther Health Med 2007;13:36-8.

21 Bogusch LM, O'Brien WH. The effects of Mindfulness-Based interventions on diabetes-related distress, quality of life, and metabolic control among persons with diabetes: a meta-analytic review. Behav Med 2019;45:19-29.

22 Klainin-Yobas P, Cho MAA, Creedy D. Efficacy of mindfulness-based interventions on depressive symptoms among people with mental disorders: a meta-analysis. Int J Nurs Stud 2012;49:109-21.

23 Kuyken W, Warren FC, Taylor RS, et al. Efficacy of MindfulnessBased cognitive therapy in prevention of depressive relapse: an individual patient data meta-analysis from randomized trials. JAMA Psychiatry 2016;73:565-74.

24 Niazi A, Niazi S. Mindfulness-based stress reduction: a nonpharmacological approach for chronic illnesses. N Am J Med Sci 2011;3:20-3.

25 Victorson D, Kentor M, Maletich C, et al. Mindfulness meditation to promote wellness and manage chronic disease: a systematic review and meta-analysis of Mindfulness-Based randomized controlled trials relevant to lifestyle medicine. Am J Lifestyle Med 2015;9:185-211.

26 Kabat-Zinn J. Wherever you go, there you are: mindfulness meditation in everyday life. New York, NY: Hyperion, 1994.

27 Noordali F, Cumming J, Thompson JL. Effectiveness of Mindfulnessbased interventions on physiological and psychological complications in adults with diabetes: a systematic review. J Health Psychol 2017;22:965-83.

28 Moher D, Liberati A, Tetzlaff J, et al. Preferred reporting items for systematic reviews and meta-analyses: the PRISMA statement. Int $J$ Surg 2010;8:336-41.

29 Dinardo MM. A mindful approach to diabetes self-management education with stress reduction and healthy coping for US veterans with diabetes. Dissertations \& Theses - Gradworks, 2013.

30 Jung HY, Lee H, Park J. Comparison of the effects of Korean mindfulness-based stress reduction, walking, and patient education in diabetes mellitus. Nurs Health Sci 2015;17:516-25. 
31 Higgins JPT, Altman DG, Sterne JAC, et al. Chapter 8: Assessing risk of bias in included studies [C]//Higgins JPT, Green S. Cochrane Handbook for Systematic Reviews of Interventions Version 5.1.0. The Cochrane Collaboration, 2011. Available: www.cochranehandbook.org

32 Cohen J. Statistical power analysis for behavioural science. 2nd ed. Hillsdale, NJ: Erlbaum, 1988.

33 Higgins JPT, Thompson SG, Deeks JJ. Measuring inconsistency in meta-analyses. BMJ 2003;327:557-60.

34 DerSimonian R, Laird N, Nan L. Meta-Analysis in clinical trials. Control Clin Trials 1986;7:177-88.

35 van Son J, Nyklíček I, Pop VJ, et al. Mindfulness-based cognitive therapy for people with diabetes and emotional problems: long-term follow-up findings from the DiaMind randomized controlled trial. $J$ Psychosom Res 2014;77:81-4.

36 Schroevers MJ, Tovote KA, Keers JC, et al. Individual MindfulnessBased cognitive therapy for people with diabetes: a pilot randomized controlled trial. Mindfulness 2015;6:99-110.

37 Tovote KA, Schroevers MJ, Snippe E, et al. Long-Term effects of individual mindfulness-based cognitive therapy and cognitive behavior therapy for depressive symptoms in patients with diabetes: a randomized trial. Psychother Psychosom 2015;84:186-7.

38 Friis AM, Johnson MH, Cutfield RG, et al. Kindness Matters: A Randomized Controlled Trial of a Mindful Self-Compassion Intervention Improves Depression, Distress, and $\mathrm{HbA}{ }_{1 \mathrm{c}}$ Among Patients With Diabetes. Diabetes Care 2016;39:1963-71.

39 LeiTen QZ. Effect of mindfulness therapy on psychological pain and quality of life in type 2 diabetes. Chinese $J$ Health Psychol 2018;26:197-200.

40 Pearson S, Wills K, Woods M, et al. Effects of mindfulness on psychological distress and $\mathrm{HbA} 1 \mathrm{c}$ in people with diabetes. Mindfulness 2018;9:1615-26.
41 Yang J, Zhao X, Kang S, et al. Effect of mindfulness therapy on psychological distress and quality of life in type 2 diabetes. Chin Med J Nurs 2018;24:186-90.

42 Schmitt A, Reimer A, Kulzer B, et al. How to assess diabetes distress: comparison of the problem areas in diabetes scale (paid) and the diabetes distress scale (DDS). Diabet Med 2016;33:835-43.

43 Hofmann SG, Sawyer AT, Witt AA, et al. The effect of mindfulnessbased therapy on anxiety and depression: a meta-analytic review. J Consult Clin Psychol 2010;78:169-83.

44 Bohlmeijer E, Prenger R, Taal E, et al. The effects of mindfulnessbased stress reduction therapy on mental health of adults with a chronic medical disease: a meta-analysis. J Psychosom Res 2010;68:539-44.

45 Segal ZV. Mindfulness based cognitive therapy for depression: a new approach to preventing relapse. New York, NY: Guilford Press, 2002.

46 Abbott RA, Whear R, Rodgers LR, et al. Effectiveness of mindfulness-based stress reduction and mindfulness based cognitive therapy in vascular disease: a systematic review and meta-analysis of randomised controlled trials. J Psychosom Res 2014;76:341-51.

47 Wang new progresses of group psychotherapy for treatment of depression. Journal of Shanghai JiaoTong University 2015;35.

48 Strauss C, Hayward M, Chadwick P. Group person-based cognitive therapy for chronic depression: a pilot randomized controlled trial. British Journal of Clinical Psychology 2012;51:345-50.

49 Parsons CE, Crane C, Parsons LJ, et al. Home practice in Mindfulness-Based cognitive therapy and Mindfulness-Based stress reduction: a systematic review and meta-analysis of participants mindfulness practice and its association with outcomes. Behav Res Ther 2017;95:29-41.

50 Stannard D. Essentials of nursing research: appraising evidence for nursing practice. Aorn J 2012;95:307-8. 